\title{
Maxwell's Equations in Divergence Form for General Media with Applications to MHD*
}

\author{
Maurice H. P. M. van Putten \\ Department of Astrophysics 130-33, Caltech, Pasadena, CA 91125, USA \\ Received October 26, 1990; in revised form April 9, 1991
}

\begin{abstract}
Maxwell's equations in media with general constitutive relations are reformulated in covariant form as a system of divergence equations without constraints. Our reformulation enables us to express general electro-magneto-fluid problems as hyperbolic systems in divergence form. We illustrate this method on the MHD problem. In the absence of constraints, a general representation is derived for the characteristic form for first-order systems of quasi-linear partial differential equations in vector fields and scalars. Using this covariant formulation of characteristics, we find that the principle of covariance imposes a very rigid structure on the infinitesimally small amplitude waves in MHD. To demonstrate the power of the reformulation, we study numerically ultra-relativistic wave breaking using the divergence formulation of MHD.
\end{abstract}

\section{Introduction}

Maxwell's equations appear in a wide variety of problem settings in general relativity. We will consider them as they appear in general relativistic formulations of electro-magneto-fluid problems. They appear in their natural form as an underdetermined system of divergence equations. Lichnerowicz [5] showed that implementation of constitutive relations of a particular medium yields a pair of scalar constraints. Thus, electromagnetic fields in general media are determined completely by a mixed partial differential-algebraic system of equations.

Numerical treatment of electro-magneto-fluid problems by standard methods requires these problems to be formulated as a system consisting purely of partial differential equations with no constraints. Of course, the constraints as they appear in Lichnerowicz's formulation are avoided when taking the electromagnetic field variables as 3 -vectors (cf. $[12,18]$ ). The electromagnetic fields in general

\footnotetext{
* This material is based upon work supported by NSF grant AST 84-51725. Some of the work has been performed at the Department of Applied Mathematics, Caltech
} 
media are then determined by a quasi-linear system of differential equations in an explicit space-time split.

In this paper, we will show that the constraints from Lichnerowicz's formulation can become conserved quantities in a new system of partial differential equations in which the electromagnetic field variables remain 4-vectors. Thus, we will arrive at a system consisting purely of partial differential equations with no constraints. We will show

Theorem 1. Maxwell's equations in general media can be reformulated in covariant form as a system of divergence equations without constraints.

Theorem 1 enables us to formulate general electro-magneto-fluid problems as hyperbolic systems in divergence form. The divergence form is well-known to be a good starting point for numerical implementation. Advanced numerical methods have been developed in classical fluid dynamics for hyperbolic systems of this form. Theorem 1 thus allows general electro-magneto-fluid problems to be approached numerically by existing numerical methods from computational fluid dynamics (see, e.g., [25]). In divergence form, it now also becomes possible to treat electro-magneto-fluid problems numerically in the weak formulation. It is well-known that weak formulations of systems in divergence form uniquely determine the jump conditions across shocks (cf. [16]). The shock structure of Maxwell's solutions in the new formulation will be discussed in detail.

To illustrate this theorem from an analytical perspective, we apply it to the classical MHD problem and show that MHD can be reformulated as a system of divergence equations without constraints. In this form, the MHD problem can now be treated numerically by any of the standard numerical methods from classical fluid dynamics.

The theorem also allows for a general formulation of the problem of characteristics for a large class of electro-magneto-fluid problems. The associated questions of hyperbolicity and wave structure are central in relativistic magnetofluid dynamics $[26,27,4-6,2,1,17]$. Our theorem permits us to formulate this problem of characteristics in terms of vector fields and scalars. We derive a general expression for the characteristic form of the associated system of partial differential equations.

To illustrate this approach, we show how the principle of covariance imposes the general structure on the infinitesimally small wave equations in MHD.

In Sect. 2, we prove the Theorem, and in Sect. 3 we discuss the shock structure of the new formulation. In Sect. 4, we reformulate MHD as a system in divergence form. We present our general theory of characteristics in Sect. 5. Our derivation of characteristics for MHD is discussed in Sect.6, and our numerical study of ultra-relativistic wave breaking is presented in Sect. 7.

\section{Maxwell's Equations in Divergence Form}

In this section we prove our Theorem, showing that Maxwell's equations in general media can be written in divergence form without constraints. Maxwell's equations can be stated in terms of a pair of divergences of 2 -forms $\mathbf{H}$, the electric field-magnetic induction tensor, and $\mathbf{G}$, the electric induction-magnetic field tensor, [5] as

$$
\begin{aligned}
\nabla^{a} * H_{a b} & =0, \\
\nabla^{a} G_{a b} & =-j_{b},
\end{aligned}
$$


where $\mathbf{j}$ is the electric current 1 -form. Here, $*$ denotes the Hodge star operator defining the dual $* \boldsymbol{\alpha}$ of a $p$-form $\mathbf{a}$ on an $n$-dimensional Riemannian manifold as

$$
* \alpha_{i_{1} \ldots i_{n-p}} \equiv \frac{1}{p !} \alpha^{j_{1} \ldots j_{p}} \varepsilon_{j_{1} \ldots j_{p} i_{1} \ldots i_{n-p}},
$$

where $\epsilon$ is the Levi-Civita tensor. Throughout this paper we use the convention that roman indices rum from 0 to 3 . The constitutive relations for a medium yield two scalar constraints. Before proceeding to prove the theorem, we show how these constraints arise.

In a medium with velocity four-vector $\mathbf{u}, u^{b} u_{b}=-1$, we have

$$
\begin{aligned}
& \left(e_{a}, b_{a}\right):=\left(u^{b} H_{a b}, u^{b} * H_{a b}\right), \\
& \left(d_{a}, h_{a}\right):=\left(u^{b} G_{a b}, u^{b} * G_{a b}\right)
\end{aligned}
$$

for the electric field, $\mathbf{e}$, magnetic induction, $\mathbf{b}$, electric induction, $\mathbf{d}$, and magnetic field, $\mathbf{h}$, respectively. We remark that as a consequence of the antisymmetry of $\mathbf{H}$ and $\mathbf{G}$, we have the algebraic identities

$$
\begin{aligned}
& u^{b} e_{b}=u^{b} h_{b}=0, \\
& u^{b} d_{b}=u^{b} b_{b}=0 .
\end{aligned}
$$

The 2-forms $\mathbf{H}$ and $\mathbf{G}$ can now be expressed as $[5,7]$

$$
\begin{aligned}
& \mathbf{H}=\mathbf{u} \wedge \mathbf{e}-*(\mathbf{u} \wedge \mathbf{b}), \\
& \mathbf{G}=\mathbf{u} \wedge \mathbf{d}-*(\mathbf{u} \wedge \mathbf{h}) .
\end{aligned}
$$

Here, the velocity four-vector $\mathbf{u}$ actually enters as its dual one-form, but we will not make this explicit.

Thus, Maxwell's equations are a set of evolution equations for the family of tensor fields $U=(\mathbf{e}, \mathbf{d}, \mathbf{h}, \mathbf{b}, \mathbf{u}, \mathbf{g}, q)$ with given $\mathbf{g}$, where $\mathbf{g}$ is the metric. The scalar variable $q$ (which corresponds to the electric charge density) arises as an extra degree of freedom so that the following familiar relationship holds:

$$
0 \equiv d^{2} * \mathbf{G}=d * \mathbf{j}
$$

Here, $d$ denotes the exterior derivative. We remark that there can be no confusion between the $d$ for the exterior derivative and that for the electric induction, because the latter always explicitly appears as a tensor.

In this form, Maxwell's equations can be closed by local constitutive relations of the form

$$
\begin{aligned}
\mathbf{d} & =\tilde{\mathbf{d}}(\mathbf{e}, \mathbf{h}, \mathbf{u}, \mathbf{g}, q), \\
\mathbf{b} & =\tilde{\mathbf{b}}(\mathbf{e}, \mathbf{h}, \mathbf{u}, \mathbf{g}, q), \\
\mathbf{j} & =\tilde{\mathbf{j}}(\mathbf{e}, \mathbf{h}, \mathbf{u}, \mathbf{g}, q)
\end{aligned}
$$

with $\partial \tilde{\mathbf{j}}(U) / \partial q \neq 0$ and such that the identities

$$
u^{a} \tilde{d}_{a}(U)=u^{a} \tilde{b}_{a}(U)=0
$$

hold as algebraic implications of (4). For example, in the familiar case of linear, isotropic media this reduces to

$$
\tilde{\mathbf{d}}(U)=\varepsilon \mathbf{e}, \quad \tilde{\mathbf{b}}(U)=\mu \mathbf{h},
$$


where $\varepsilon$ is the electric permittivity and $\mu$ is the magnetic permeability. Furthermore, using the fact that $\mathbf{u}$ is nowhere vanishing, it is consistent to take

$$
\tilde{\mathbf{j}}(U)=q \mathbf{u}+\sigma \mathbf{e}
$$

with $\sigma$ as the electric conductivity. In this case, $q$ is precisely the electric charge density.

As a result, Maxwell's equations are stated as a set of evolution equations for $V=(\mathbf{e}, \mathbf{h}, q)$ (and $\mathbf{u})$ in the family of variables $V=(V, \mathbf{u}, \mathbf{g})$ as a mixed partial differential-algebraic system of equations as [5]

$$
\left\{\begin{aligned}
\nabla^{a} * H_{a b}(U) & =0 \\
\nabla^{a} G_{a b}(U) & =-\tilde{j}_{b}(U), \\
c_{1}(U):=u^{a} h_{a} & =0 \\
c_{2}(U):=u^{a} e_{a} & =0 .
\end{aligned}\right.
$$

This comprises a set of 10 equations for the 9 variables $V$. In the degenerate case of MHD when the medium is linear, isotropic with $\sigma$ infinite, this reduces to

$$
\left\{\begin{aligned}
\nabla^{a} u_{[a} b_{b]} & =0, \\
u^{a} b_{a} & =0,
\end{aligned}\right.
$$

in view of $\mathbf{e} \equiv \mathbf{d} \equiv 0$. This comprises a set of 5 equations for the four unknowns b.

The sets of equations above evidently consist of systems of the type

$$
K:\left\{\begin{aligned}
\nabla^{a} \omega_{a b} & =j_{b}, \\
c & =0,
\end{aligned}\right.
$$

where $\omega$ is a 2 -form, $\mathbf{j}$ is a 1 -form, and $c=0$ forms a scalar constraint. Now consider a Cauchy-problem for $K$ on a smooth space-like hypersurface $\Sigma$ in a hyperbolic Riemannian space (M, $\mathbf{g}$ ) with given metric $\mathbf{g}$. Cauchy-data for $K$ must satisfy a compatibility condition. This can be made precise as follows. Let $\mathbf{v}$ be a unit vector field normal to $\Sigma$. Decomposing $\nabla$ on the space-like $\Sigma$ orthogonally as

$$
\nabla_{a}=-v_{a}\left(v^{c} \nabla_{c}\right)+\left(\nabla_{\Sigma}\right)_{a}
$$

where $\nabla_{\Sigma}$ is interior to $\Sigma$, we can rewrite $K$ on $\Sigma$ as

$$
-v^{a}\left(v^{c} \nabla_{c}\right) \omega_{a b}+\left(\nabla_{\Sigma}\right)^{a} \omega_{a b}=j_{b} .
$$

Next, we observe that

$$
v^{a} v^{b}\left(v^{c} \nabla_{c}\right) \omega_{a b} \equiv 0,
$$

because $\boldsymbol{\omega}$ is antisymmetric. Therfore, the Cauchy-data on $\Sigma$ must satisfy the two compatibility conditions

$$
\left\{\begin{aligned}
v^{b}\left(\left(\nabla_{\Sigma}\right)^{a} \omega_{a b}-v^{b} j_{b}\right) & =0, \\
c & =0 .
\end{aligned}\right.
$$

We have, in the context of classical $C^{2}(\mathbf{M})$ solutions,

Lemma 2.1. A Cauchy-problem for $K$ on $\Sigma$ can be reformulated as a Cauchyproblem for

$$
K^{\prime}:\left\{\begin{aligned}
\nabla^{a}\left(\omega_{a b}+g_{a b} c\right) & =j_{b}, \\
\nabla^{a} j_{a} & =0
\end{aligned}\right.
$$


on $\Sigma$ with the same Cauchy-data in the sense that if a solution exists to one then it exists to the other and the solutions agree in the future domain of dependence of $\Sigma$.

Proof. Clearly, we need only show that a classical solution to the new formulation with Cauchy-data compatible with $K$ yields a classical solution to the original $K$-formulation. We will do so by showing that $c$ satisfies the canonical wave equation with vanishing Cauchy-data:

$$
\begin{cases}\square c=0 & \text { in } D^{+}(\Sigma), \\ c=0 & \text { on } \Sigma, \\ v^{a} \nabla_{a} c=0 & \text { on } \Sigma .\end{cases}
$$

Here, $\square=g^{a b} \nabla_{a} \nabla_{b}$ is the Laplace-Beltrami wave operator [8], $D^{+}(\Sigma)$ denotes the future domain of dependence of $\Sigma$ (cf. $[20,8]$ ), and $\mathbf{v}$ is a vector field normal to $\Sigma$. This can be derived in two steps.

Step (a). Recall that for $p$-forms $\mathbf{a}$ on Riemannian manifolds the following identity holds [1]:

$$
(p-1) !(-1)^{p+1} *^{-1} d * \mathbf{\alpha}=\nabla^{a} \alpha_{a i_{1} \ldots i_{p-1}} \mathbf{d} \mathbf{x}^{1} \wedge \cdots \wedge \mathbf{d} \mathbf{x}^{p-1} .
$$

Consequently, we have

$$
\nabla^{b} \nabla^{a} \omega_{a b}=*^{-1} d^{2} * \omega=0 .
$$

Therefore, $\nabla^{b} j_{b}=0$ implies

$$
0=\nabla^{b}\left(\nabla^{a}\left(\omega_{a b}+g_{a b} c\right)-j_{b}\right)=g^{a b} \nabla_{a} \nabla_{b} c \equiv \square c .
$$

Step (b). Now consider a classical solution to the new formulation with Cauchydata on $\Sigma$ which satisfy the compatibility conditions for $K$. Then using

$$
\nabla_{a}=-v_{a}\left(v^{c} \nabla_{c}\right)+\left(\nabla_{\Sigma}\right)_{a}
$$

as before, we have

$$
\begin{aligned}
0 & =v^{b}\left(\nabla^{a}\left(\omega_{a b}+g_{a b} c\right)-j_{b}\right) \\
& =-v^{b} v^{a}\left(v^{c} \nabla_{c}\right) \omega_{a b}+v^{b}\left(\left(\nabla_{\Sigma}\right)^{a} \omega_{a b}-j_{b}\right)+v^{b} \nabla_{b} c \\
& =v^{b} \nabla_{b} c,
\end{aligned}
$$

because $\boldsymbol{\omega}$ is antisymmetric.

Together, Step (a) and Step (b) show that $c$ satisfies the wave equation with vanishing Cauchy-data. This forces $c \equiv 0$ in $D^{+}(\Sigma)$ (cf. $[20,8]$ ), and the proof is complete.

This allows us to obtain Maxwell's equations in precisely the number of variables in $V$, because the lemma directly yields:

Theorem 2.1. The equations of Maxwell can be reformulated as a system of divergence equations as

$$
\left\{\begin{aligned}
\nabla^{a}\left(* H_{a b}+g_{a b} c_{1}\right)(U) & =0, \\
\nabla^{a}\left(G_{a b}+g_{a b} c_{2}\right)(U) & =-\tilde{j}_{b}(U), \\
\nabla^{a} \tilde{j}_{a}(U) & =0
\end{aligned}\right.
$$

in the sense as described in the lemma. 
The constraints in Maxwell's equations have thus been given a conservative implementation. We emphasize that the new formulation imposes no compatibility conditions on the Cauchy-data on $\Sigma$. With arbitrary Cauchy-data we may construct solutions to the new formulation in which $c$ is no longer vanishing. It is only when the compatibility conditions for $K$ are satisfied that, as we have shown above, $c$ will remain zero, and the solution will be a Maxwell's solution. In the case of a charged fluid a solution with $c_{2}(U)=u^{a} e_{a} \neq 0$ leads to forces along world lines. For this reason, solutions with $c_{1}, c_{2} \neq 0$ will be regarded as nonphysical.

In this sense the new formulation features a larger class of solution than the original formulation of Maxwell's equations. Therefore, a detailed discussion of Maxwell's solutions with shocks in the new formulation is required.

\section{Shock Structure}

We will discuss the shock structure of the new formulation of Maxwell's equations in terms of $K^{\prime}$. Consider a solution to $K^{\prime}$ which possesses a smooth, time-like shock surface $S$. Let $\mathbf{v}$ denote a vector field normal to $S$. Then the following jump conditions must hold

$$
\left\{\begin{array}{l}
0=v^{a}\left[\omega_{a b}+g_{a b} c\right]=v^{a}\left[\omega_{a b}\right]+v_{b}[c], \\
0=v^{a}\left[j_{a}\right] .
\end{array}\right.
$$

Here, $[f]=(f)^{+}-(f)^{-}$denotes the jump across $S$. Consequently,

$$
0=v^{a} v_{a}[c]
$$

by antisymmetric of $\boldsymbol{\omega}$, and hence of [ $\boldsymbol{\omega}]$. Since $S$ is not null, it follows that

$$
[c]=0 \text {. }
$$

Thus, we obtain

Lemma 3.1. The jump conditions for $K$ across a smooth shock surface $S$,

$$
\left\{\begin{aligned}
v^{a}\left[\omega_{a b}\right] & =0, \\
v^{a}\left[j_{a}\right] & =0,
\end{aligned}\right.
$$

are preserved in the new formulation $K^{\prime}$.

Now consider an open neighborhood $\Omega$ of $S$. Let $\Omega^{-}$and $\Omega^{+}$denote the subregions of $\Omega$ lying at either side of $S$. Let $I^{-}(S)$ denote the chronological past of $S$ (cf. [20]). We have

Lemma 3.2. A solution to $K^{\prime}$ in $\Omega$ which satisfies $K$ in $\Omega^{-}$is a solution to $K$ in $\Omega \cap I^{-}(S)$.

Proof. We consider a solution to $K^{\prime}$ which is $C^{2}$ in each of $\Omega^{+} \cup S$ and $\Omega^{-} \cup S$. Notice that this forces

$$
c \equiv 0 \quad \text { in } \Omega^{-} .
$$

We will show that $c$ satisfies the canonical wave equation with vanishing Cauchydata in $\Omega^{+}$:

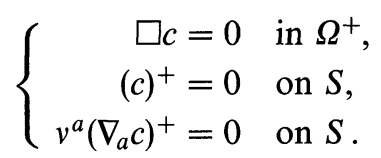


Of course, we have already demonstrated in the proof of Lemma 2.1 that $c$ satisfies the wave equation in $\Omega^{+}$. It remains to derive the Cauchy-data. We will do so in two steps.

Step (a). From the discussion preceding Lemma 3.1, we have

$$
0=[c]=(c)^{+}-(c)^{-}=(c)^{+},
$$

since $c \equiv 0$ in $\Omega^{-}$.

Step (b). Decompose $\nabla$ on $S$ as

$$
\nabla_{a}=v_{a}\left(v^{c} \nabla_{c}\right)+\left(\nabla_{S}\right)_{a} .
$$

By Lemma 3.1 and smoothness of $S$, we have

$$
0=\left(\nabla_{S}\right)^{a} v^{b}\left[\omega_{a b}\right]=v^{b}\left(\nabla_{S}\right)^{a}\left[\omega_{a b}\right]+\left[\omega_{a b}\right]\left(\nabla_{S}\right)^{a} v^{b}
$$

Let $\left\{x^{\alpha}\right\}_{\alpha=1}^{3}$ denote a coordinate system for $\Sigma$, and $\mathbf{e}_{(\alpha)}=\partial / \partial x^{\alpha}$. By Lemma 3.1, $v^{a}\left[\omega_{a b}\right]=0$ so that

$$
\left[\omega_{a b}\right]\left(\nabla_{S}\right)^{a} v^{b}=\left[\omega_{\alpha \beta}\right] \nabla^{\alpha} v^{\beta} .
$$

The symmetry of the extrinsic curvature tensor, $\mathbf{K}$, [11] in

$$
-\nabla_{\alpha} v^{\beta}=\mathbf{K}\left(\mathbf{e}_{(\alpha)}, \mathbf{e}_{(\beta)}\right) \mathbf{v}
$$

further implies

$$
\left[\omega_{\alpha \beta}\right] \nabla^{\alpha} v^{\beta}=0
$$

We, therefore, have

$$
v^{b}\left(\nabla_{S}\right)^{a}\left[\omega_{a b}\right]=0 .
$$

This and the second jump condition from Lemma 3.1 together imply

$$
\begin{aligned}
0 & =v^{b}\left(\nabla_{S}\right)^{a}\left[\omega_{a b}\right]-v^{b}\left[j_{b}\right] \\
& =v^{b}\left(\left(\nabla_{S}\right)^{a}\left(\omega_{a b}\right)^{+}-\left(j_{b}\right)^{+}\right)-v^{b}\left(\left(\nabla_{S}\right)^{a}\left(\omega_{a b}\right)^{-}-\left(j_{b}\right)^{-}\right) \\
& =v^{b}\left(\left(\nabla_{S}\right)^{a}\left(\omega_{a b}\right)^{+}-\left(j_{b}\right)^{+}\right),
\end{aligned}
$$

since the solution to $K^{\prime}$ is assumed to be a $C^{2}$ solution to $K$ in $\Omega^{-} \cup S$. Therefore, the solution to $K^{\prime}$ satisfies

$$
\begin{aligned}
0 & =v^{b}\left(\nabla^{a}\left(\omega_{a b}+g_{a b} c\right)-j_{b}\right)^{+} \\
& =v^{b}\left(\left(\nabla_{S}\right)^{a}\left(\omega_{a b}\right)^{+}-\left(j_{b}\right)^{+}\right)+v^{b}\left(\nabla_{b} c\right)^{+} \\
& =v^{b}\left(\nabla_{b} c\right)^{+} .
\end{aligned}
$$

We remark that a proof for this result in a weak formulation can also be given.

Together, Step (a) and Step (b) show that $c$ satisfies the canonical wave equation in $\Omega^{+}$with vanishing Cauchy-data. This forces $c \equiv 0$ in $\Omega \cap I^{-}(S)$ by Holmgren's Uniqueness Theorem (cf. [23]).

We have therefore demonstrated

Proposition 3.1. Maxwell's solutions are preserved across smooth shock surfaces in the divergence formulation of Theorem 2.1 in the sense as described in Lemma 2.1. 


\section{MHD in Divergence Form}

In this section, ideal MHD is formulated as a system of divergence equations with no constraints. Consider a perfectly conducting fluid with unit velocity fourvector $\mathbf{u}$ in a background with metric $\mathbf{g}$. The fluid is described by a stress-energy tensor [9]

$$
\mathbf{T}^{M}=r f \mathbf{u} \otimes \mathbf{u}+P \mathbf{g},
$$

where $r$ is the proper restmass density, $f$ is the specific enthalpy and $P$ is the fluid pressure. Physically, $f$ appears as $f=f(r, S)$ with entropy $S$. However, one usually takes $r=r(f, S)$ in view of

$$
d P=r d f-r T d S
$$

as the implicit definition of the temperature $T$. The electromagnetic field is described by

$$
\mathbf{T}^{E M}=\mathbf{b}^{2}(\mathbf{u} \otimes \mathbf{u}+\mathbf{g} / 2)-\mathbf{b} \otimes \mathbf{b},
$$

where $\mathbf{b}$ is the magnetic induction. Write

$$
\mathbf{T}=\mathbf{T}^{M}+\mathbf{T}^{E M}
$$

for the total stress energy tensor. The standard governing equations for MHD comprise a mixed partial differential-algebraic system of equations of the form $[26,5,6,19]$

$$
\left\{\begin{aligned}
\nabla^{a} T_{a b} & =0, \\
\nabla^{a} u_{[a} b_{b]} & =0, \\
\nabla^{a}\left(r u_{a}\right) & =0, \\
u^{a} \nabla_{a} S & =0, \\
u^{a} b_{a} & =0, \\
u^{a} u_{a} & =-1 .
\end{aligned}\right.
$$

It is well-known that $u^{a} u_{a}=-1$ is conserved along streamlines, so that MHD is a problem with essentially one constraint: $u^{a} b_{a}=0$. Anile and Pennisi [19] reformulated this standard form of MHD as a quasi-linear system of partial differential equations. They obtained their result by a detailed study of the equations. However, their final system is not in divergence form.

Our theorem allows us to obtain:

Corollary 4.1. The equations of ideal MHD can be reformulated as a system of divergence equations without constraints as

$$
\left\{\begin{aligned}
\nabla^{a} T_{a b} & =0, \\
\nabla^{a}\left(u_{[a} b_{b]}+g_{a b} u^{c} b_{c}\right) & =0, \\
\nabla^{a}\left(r u_{a}\right) & =0, \\
\nabla^{a}\left(r S u_{a}\right) & =0
\end{aligned}\right.
$$

in the sense as described in the lemma.

In ths form, MHD may now be treated numerically by any of the standard numerical methods for hyperbolic systems in divergence form. To illustrate one of its analytical aspects, we will show that this formulation will naturally yield the well-known characteristics for MHD. To this end, we first put the problem of characteristics in a more general setting. 


\section{A Covariant Formulation of Characteristics}

A large class of electro-magneto-fluid problems in general relativity can be formulated in terms of a family of tensor fields $U=\left(a^{1}, \ldots, a^{p}, \mathbf{u}^{1}, \ldots, \mathbf{u}^{q}, \mathbf{g}\right)$ which consists entirely of scalars $a^{i} \in \mathscr{T}_{0}^{0}(\mathbf{M})$, vector fields $\mathbf{u}^{j} \in \mathscr{T}_{0}^{1}(\mathbf{M})$ and a hyperbolic metric $\mathbf{g} \in \mathscr{T}_{2}^{0}(\mathbf{M})$. Here, $\mathscr{T}_{s_{i}}^{r_{i}}(\mathbf{M})$ are the tensor fields of type $\left(r_{i}, s_{i}\right)$ on a four-dimensional manifold $\mathbf{M}$. Our theorem enables us to formulate Maxwell's equations as a system consisting only of partial differential equations with no constraints. For this reason, the class of problems that we will discuss are those for which the evolution equations for a subset $V=\left(a^{1}, \ldots, a^{p^{\prime}}, \mathbf{u}^{1}, \ldots, \mathbf{u}^{q^{\prime}}\right)$ $\left(p^{\prime} \leq p, q^{\prime} \leq q\right)$ of $U$ can be expressed in the general form

$$
A(U ; \nabla) V=f(U) \text {. }
$$

Here, $A(U ; \nabla): \mathscr{Y}(\mathbf{M}):=\left(\mathscr{T}_{0}^{0}(\mathbf{M})\right)^{p^{\prime}} \times\left(\mathscr{T}_{0}^{1}(\mathbf{M})\right)^{q^{\prime}} \rightarrow \mathscr{Y}(\mathbf{M})$ is a local, first order quasi-linear differential operator and $f(U)$ is local in $V$.

The problem of characteristics can be stated in terms of a Cauchy problem in an open neighborhood $\mathcal{N}(\Sigma)$ of a 3-dimensional initial manifold $\Sigma$ with prescribed Cauchy-data $U^{0}$ as

$$
C: \begin{cases}A(U ; \nabla) V=f(U) & \text { in } \mathscr{N}(\Sigma), \\ U=U^{0} & \text { on } \Sigma .\end{cases}
$$

The initial hypersurface $\Sigma$ is now said to be characteristic whenever $U^{0}$ on $\Sigma$ and $U^{\prime}=\left(\mathbf{u}^{q^{\prime}+1}, \ldots, \mathbf{u}^{q}, \mathbf{g}\right)$ in $\mathcal{N}(\Sigma)$ do not suffice to obtain $V$ away from $\Sigma$ into $\mathscr{N}(\Sigma)$. We will study this problem pointwise in $\mathbf{M}$ as a function of the orientation of nonnull $\Sigma$ in $(\mathbf{M}, \mathbf{g})$. The case when $\Sigma$ is null is excluded specifically. Nullcharacteristic hypersurfaces form an intricate problem that we will not touch upon here. For this problem we refer to Muller zum Hagen and Seifert [28] and references therein.

Nonnull characteristics can be defined as follows. We first decompose $\nabla$ as $\nabla_{a}= \pm v_{a}\left(v^{c} \nabla_{c}\right)+\left(\nabla_{\Sigma}\right)_{a}$ on $\Sigma$, depending on whether $\Sigma$ is time-like ( +$)$ or space-like (-). We obtain $C$ in "Cauchy-Kowalewski" form on $\Sigma$ as:

$$
\pm A\left(U^{0} ; \mathbf{v}\right)\left(v^{a} \nabla_{a}\right) V+a\left(U^{0} ; \nabla_{\Sigma}\right) V=f(U) .
$$

The characteristic hypersurfaces are then defined by nonnull $\mathbf{v}$ such that $A(U ; \mathbf{v})(p)$ is not invertible as a map from the bundle $Y(p):=\left(T_{0}^{0}(p)\right)^{p^{\prime}} \times\left(T_{0}^{1}(p)\right)^{q^{\prime}}$ into itself $(p \in \Sigma)$. Let us use the standard covariant definition of the determinant of $A\left(U^{0} ; \mathbf{v}\right)(p)$ [15, p. 79], $\operatorname{det} A\left(U^{0} ; \mathbf{v}\right)(p)$, to define

$$
\left(\operatorname{det} A\left(U^{0} ; \mathbf{v}\right)\right)(p)=\operatorname{det} A\left(U^{0} ; \mathbf{v}\right)(p)(p \in \mathbf{M}) .
$$

Thus, there exists a natural, covariant definition for the determinant of $A(U ; \mathbf{v})$ as a scalar field on $\Sigma$. The condition for $\Sigma$ to be characteristic therefore becomes

$$
\operatorname{det} A\left(U^{0} ; \mathbf{v}\right)=0 \text {. }
$$

Such a scalar field possesses a very rigid dependence on its arguments. We have the following general representation for the characteristic form of (28):

Proposition 5.1. Let $U$ be as above. If $V=\left(a^{1}, \ldots, a^{p^{\prime}}, \mathbf{u}^{1}, \ldots, \mathbf{u}^{q^{\prime}}\right)\left(p^{\prime} \leq p, q^{\prime} \leq q\right)$ then

$$
\operatorname{det} A(U ; \mathbf{v})=\sum a_{\mu_{1} \ldots \mu_{q^{\prime} \gamma}}(U)\left(u_{a}^{1} v^{a}\right)^{\mu_{1}} \ldots\left(u_{a}^{q^{\prime}} \nu^{a}\right)^{\mu_{q}^{\prime}}\left(v_{a} v^{a}\right)^{\gamma},
$$

where $\mu_{1}+\cdots+\mu_{q^{\prime}}+2 \gamma=p^{\prime}+4 q^{\prime}$ and the $a_{\mu_{1} \ldots \mu_{q^{\prime}}}(U)$ are local scalars in $U$. 
A formal proof of this fact is not difficult but somewhat lengthy. We will merely remark that the basic mathematical ingredients are locality and the theorems of Stone-Weierstrass and Riesz. Using these, the proposition follows from invariance of the physics under diffeomorphisms $\phi: \mathbf{M} \rightarrow \mathbf{M}$. If $\phi^{*}$ denotes the pull forward map associated with such $\phi$, it suffices to consider the invariances

(i) $\operatorname{det} A(U ; \mathbf{v})(p)=\operatorname{det} A \circ \phi^{*}(U ; \mathbf{v})(p), \quad \phi(p)=p$,

(ii) $\operatorname{det} A(U ; \mathbf{v})(p)=\operatorname{det} A \circ \phi^{*}(U ; \mathbf{v})\left(\phi^{-1}(p)\right), \quad \nabla \phi(p)=\mathrm{id}$.

The first invariance is known as scalar invariance and isotropy, and the second invariance is known as homogeneity.

We wish to emphasize the following. The set of zeros $\mathbf{v}_{p} \in \mathscr{T}_{1}^{0}(p)$ for which $A\left(U^{0} ; \mathbf{v}\right)(p)$ is singular is called the normal cone at $p \in \mathbf{M}[13]$. This normal cone contains the normals to the characteristic hypersurfaces. These characteristic hypersurfaces carry the infinitesimally small amplitude waves. As such, these zeros must be invariant by the principle of covariance. Thus, if we somehow knew that the normal cone possesses a full set of $N$ real sheets of zeros we could say that this suffices to establish the covariant expression (33), up to a nonzero factor. However, in the general case or, more importantly, in proving that the system possesses a full set of $N$ real, spacelike sheets (algebraic hyperbolicity), we need to go through the full formal arguments above.

A system of partial differential equations is regular in the sense of CauchyKowalewski if its characteristic form is not identically zero [11]. Systems involving Maxwell's equations (13) without incorporation of the constraints are not regular in this sense. Systems impose uniqueness, and, therefore, may be a given direct numerical implementation, only when regular in the sense of Cauchy-Kowalewski. It is easy to see that the divergence formulation (18) regularizes Maxwell's equations (13). This is also exemplified in Sect. 5.

The usefulness of such a general form lies in the possibility of a priori partial factorization by using elementary facts about the problem at hand. The problem can be considered in terms of blocks $A^{(i)}(U ; \nabla)$ each corresponding to a specific subset of tensors from $V$. Considering these blocks individually, we can consider the rank of each of them. Usually, ther will be one or more blocks with known degeneracies, i.e., simple scalar conditions which imply a change in the rank of $A^{(i)}\left(U^{0} ; \mathbf{v}\right)$, and a vanishing of $\operatorname{det} A\left(U^{0} ; \mathbf{v}\right)$. By Proposition 5.1, we can then arrive at a partial factorization of $\operatorname{det} A\left(U^{0} ; \mathbf{v}\right)$. This will be illustrated in our treatment of ideal MHD. We will call this the method of uncoupled factors. This can result in a dramatic reduction of the characteristic determinant. This completes our covariant implementation of constraints in the problem of characteristics for problems of type $C$.

\section{Infinitesimally Small Amplitude Waves in MHD}

The structure of the infinitesimally small amplitude waves will follow from the equations of the characteristics. The characteristics for MHD in Corollary 4.1 as given by Proposition 5.1 are in $V=(\mathbf{u}, \mathbf{b}, f, S)$ and $U=(V, \mathbf{g})$. The evolution of the metric $\mathbf{g}$ is described by the Einstein equations

$$
\mathbf{G}=8 \pi \mathbf{T},
$$

where the Einstein tensor $\mathbf{G}$ depends on $\mathbf{g}$ (up to its second derivatives) only, while $\mathbf{T}$ is a function of all the tensor fields but none of its derivatives. Furthermore, 
in MHD only $\mathbf{g}$ and none of its derivatives appear. Thus, the problem of characteristics for the Einstein equations is independent of that of MHD. We will consider the problem of characteristics of MHD only. Alternatively, we could say that we consider MHD in the background of a given, fixed metric.

By Proposition 5.1, we have in this case

$$
\operatorname{det} A(U ; \mathbf{v})=D_{N}(U ; \mathbf{v}) \equiv \sum \alpha_{k}(U)\left(u^{a} v_{a}\right)^{v_{k}}\left(b^{a} v_{a}\right)^{\mu_{k}}\left(v^{a} v_{a}\right)^{\gamma_{k}},
$$

where $v_{k}+\mu_{k}+2 \gamma_{k}=N$ with $N=10$. Let us apply the method of uncoupled factors to partially factorize this polynomial in $\mathbf{v}$. Write $u^{a} \nabla_{a} S$ in CauchyKowalewski form on a time-like hypersurface $\Sigma$ as

$$
u^{a} v_{a}\left(v^{c} \nabla_{c}\right) S+u^{a}\left(\nabla_{\Sigma}\right)_{a} S=0 .
$$

Thus, $\left(0,0,0, u^{a} v_{a}\right)$ implements $u^{c} \nabla_{c} S$ in $A(U ; \mathbf{v})$. Clearly, its rank is zero whenever $u^{a} v_{a}=0$. Notice that $\operatorname{det} A(U ; \mathbf{v})$ is even in $\mathbf{b}$, and hence also in $\mathbf{u}$, by invariance under rotation about $\mathbf{u}$ ( $\mathbf{b}$ is space-like, $\mathbf{u}$ is time-like and $u^{a} b_{a}=0$ ). Indeed, if $\operatorname{det} A(U ; \mathbf{v})$ where odd in $\mathbf{b}$, then in going from $\mathbf{b}$ to $-\mathbf{b}$ by rotation about u det $A(U ; \mathbf{v})$ would always have a zero, forcing this to be zero for all b. But then $\operatorname{det} A(U ; \mathbf{v})$ is also even in $\mathbf{b}$. From this we have $\left(u^{a} v_{a}\right)^{2}$ as a factor. Another uncoupled factor is $v^{a} v_{a}$ due to Maxwell's equations (see the proof of Lemma 2.1). Thus, it must be that

$$
\operatorname{det} A(U ; \mathbf{v})=\left(u^{a} v_{a}\right)^{2}\left(v^{a} v_{a}\right) D_{6}(U ; \mathbf{v}) .
$$

By the evenness of $D_{6}$ in $\mathbf{u}$ and $\mathbf{b}$ we have

$$
D_{6}(U ; \mathbf{v})=R\left(\left(u^{a} v_{a}\right)^{2},\left(b^{a} v_{a}\right)^{2}, v^{a} v_{a} ; U\right) \text {. }
$$

But then $R$ is a homogeneous cubic (in its first three arguments), so that

$$
R=P Q,
$$

where $P$ is linear and $Q$ is homogeneous quadratic. Clearly, each term in $\operatorname{det} A(U ; \mathbf{v})$ contains $\left(u^{a} v_{a}\right)^{i}\left(b^{a} v_{a}\right)^{j}$ with $i+j \geq 4$, as follows by inspection of Maxwell's equations. We have, therefore,

$$
\begin{aligned}
P= & p_{1}\left(u^{a} v_{a}\right)^{2}+p_{2}\left(b^{a} v_{a}\right)^{2} \\
Q= & q_{1}\left(u^{a} v_{a}\right)^{4}+q_{2}\left(u^{a} v_{a}\right)^{2}\left(b^{a} v_{a}\right)^{2}+q_{3}\left(b^{a} v_{a}\right)^{4} \\
& +q_{4}\left(u^{a} v_{a}\right)^{2}\left(v^{a} v_{a}\right)+q_{5}\left(b^{a} v_{a}\right)^{2}\left(v^{a} v_{a}\right) .
\end{aligned}
$$

A mere inspection of the equations now yields the full expressions for the coefficients $p_{i}$. It is easy to see that the block $A^{(1,2)}(U ; \mathbf{v})$ in $A(U ; \mathbf{v})$ associated with $(\mathbf{u}, \mathbf{b})$ is of the form

$$
A^{(1,2)}(U ; \mathbf{v})=\left[\begin{array}{cccc}
\left(r f^{2}+b^{2}\right) u^{a} v_{a} & -b^{a} v_{a}+\mathbf{w} & * & * \\
\mathbf{b} \otimes \mathbf{v}-b^{a} v_{a} & u^{a} v_{a}-\mathbf{u} \otimes \mathbf{v} & * & *
\end{array}\right],
$$

where $\boldsymbol{\omega}$ is a linear combination of tensor products from $\mathbf{u}, \mathbf{b}$ and $\mathbf{v}$. Let $\mathbf{e} \neq 0$ such that $e^{a} u_{a}=e^{a} b_{a}=e^{a} v_{a}=0$. Then $e^{a} \omega_{a b}=0$. Consideration of $(\lambda \mathbf{e}, \mu \mathbf{e}, 0,0)$ as a nullvector of $A^{(1,2)}(U ; \mathbf{v})$ yields immediately

$$
P(U ; \mathbf{v})=\left(u^{a} v_{a}\right)^{2}\left(r f+b^{2}\right)-\left(b^{a} v_{a}\right)^{2} .
$$

The expression for $Q$ is now obtained by straightforward identification, for example by using a symbolic manipulator. Here, Cauchy-Kowalewski regularity 
is essential. We have used Macsyma for this purpose and thus rederived the well-known result,

$$
\begin{aligned}
Q(U ; \mathbf{v})= & (f \partial r / \partial f-r)\left(u^{a} v_{a}\right)^{4}-\left(r+b^{2} \partial r / \partial f r^{-1}\right)\left(v^{a} v_{a}\right)\left(u^{a} v_{a}\right)^{2} \\
& +r^{-1}\left(v^{a} v_{a}\right)\left(b^{a} v_{a}\right)^{2} .
\end{aligned}
$$

We should mention in this context that Macsyma is actually able to give the full factorization at once, when the problem is stated in a specific frame of reference with $\mathbf{v}$ variable. This is quite surprising, considering the fact that we are dealing here with a tenth order polynomial.

The characteristic condition (32) thus yields

Proposition 6.1 (Bruhat). MHD possesses two kinds of waves,

(i) Alfven waves: $\quad P=0$,

(ii) hydrodynamical waves: $Q=0$.

It should be mentioned that Bruhat $[26,27]$ gave the general result with variable metric which included gravitational waves. This result was derived from a detailed study of the differential equations. We wish to emphasize that the result is largely determined by the principle of covariance. This completes our discussion of waves in ideal MHD.

\section{On the Numerical Implementation}

An electro-magneto-hydrodynamic (EMHD) problem in a given background metric is completely described by Maxwell's equations and the equations of motion,

$$
\nabla_{a} T^{a b}=f^{b} .
$$

Here, $T^{a b}$ is the stress-energy tensor and $f^{b}$ is the force density four-vector. The divergence formulation of Maxwell's equations given in Theorem 1 thus enables us to formulate general EMHD problems as hyperbolic systems in divergence form.

A large class of numerical methods exists to treat problems in classical fluid dynamics for such systems in divergence form (see, e.g., [25]). Highly sophisticated schemes have been designed for the computation of solutions of problems in $1 D$ and higher dimensions with shocks (see, e.g., $[24,22,3,14]$ ). Our reformulation of EMHD problems to systems in standard form thus allows us to exploit existing numerical methods.

We will give a preliminary demonstration of this advantage below. We will discuss a $1 D$ ultra-relativistic MHD problem until shocks form. We are currently working on a $2 D$ EMHD problem, on which we expect to report in a subsequent paper.

\subsection{An Ultra-Relativistic Example}

We have computed the wave breaking problem for isentropic, transverse MHD in flat space-time. Consideration of simple waves allows for an exact error analysis, since one can compare with an exact, analytical solution. It can easily be shown that the equations for simple waves may be cast in characteristic form as

$$
\left(u^{a} \pm \alpha^{-1} \beta v^{a}\right) \nabla_{a}(\phi \pm \lambda)=0,
$$




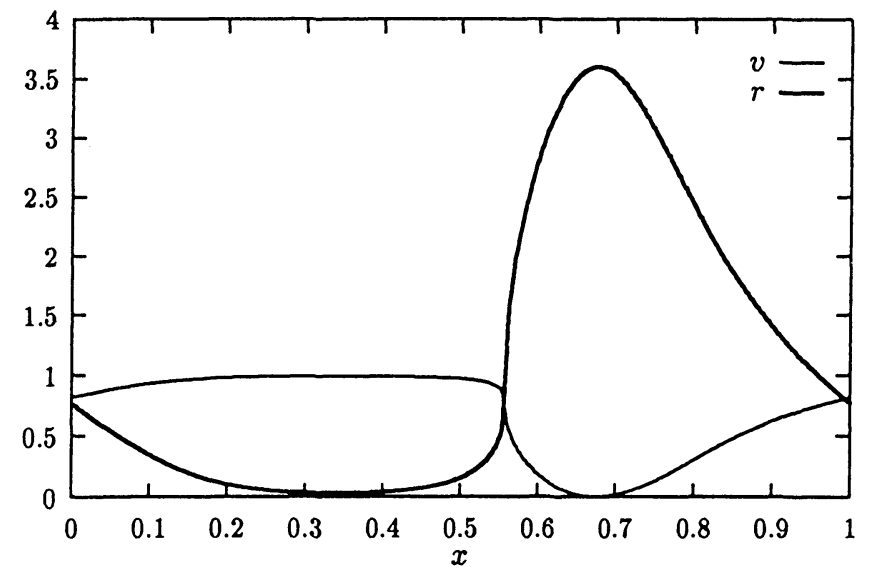

Fig. 1. The velocity distribution, $v$, and density distribution, $r$, at the moment of breaking $t=t_{B}$. In this example, $\lambda_{0}=\lambda_{1}=7 / 5, J=4.5$ and $t_{B}=0.0963$

where $u^{a}=(\cosh \lambda, \sinh \lambda, 0), v^{a}=\mathrm{d} u^{a} / \mathrm{d} \lambda, \alpha^{2}=r f^{-1} \mathrm{~d} r / \mathrm{d} f, \beta^{2}=\left(1+k^{2} \mathrm{~d} r / \mathrm{d} f\right) /$ $(1+r / f), \phi(r)=\int^{r} \alpha^{-1} \beta r^{-1}$ and $k=h / r$, which is constant throughout the fluid. Recall that a monatomic relativistic gas is described by a polytropic equation of state,

$$
P=K r^{\gamma},
$$

with polytropic index $\gamma$ between $4 / 3$ (ultra-relativistic limit) and 5/3 (Newtonian limit). In the intermediate case of $\gamma=3 / 2$, we find $\alpha^{-1} \beta(r)=\tanh \phi / 4$ when $k=1$ and $K=2 / 3$.

Our numerical example concerns a fluid of this type with the Riemann invariant $J=\lambda+\phi$ constant throughout the fluid, and $\gamma=3 / 2$. The characteristics along which the solution remains constant are then given by $\mathrm{d} x / \mathrm{d} t=\Lambda=$ $\tanh (5 \lambda / 4-J / 4)$. With initial data $\lambda(x)=\lambda_{0}+\lambda_{1} \sin 2 \pi x$, the breaking time is: $t_{B}=\inf (-\mathrm{d} \Lambda / \mathrm{d} x)^{-1}$ (see [21]) for a general discussion on breaking times).

The divergence formulation of MHD can be implemented directly using the leapfrog Crank-Nicholson method, until the shock forms. This scheme has second order accurary, provided that the solution remains smooth. Since the problem has been reformulated in standard form, we expect that the more advanced methods cited before will allow for the computation of solutions in the presence of shocks.

We have computed wave breaking problems in the Newtonian limit, in the relativistic case and in the ultra-relativistic case. We give here results only on the traditionally most difficult case, the ultra-relativistic wave breaking. Figure 1 shows the density and velocity distributions at the moment of breaking in a case when the Lorentz factor $\Gamma \approx 8$. Here, $\Gamma=1 /\left(1-v^{2}\right)^{1 / 2}$, where $v$ is the maximum velocity. In Fig. 1 the velocity of light is normalized to unity. These results have been obtained without any stabilization process. The numerical and analytical solution agree to within less than the width of the lines in the figure.

The performance of our numerical implementation is studied by the dependency of the results on the grid size $\Delta t$ in time and the grid size $\Delta x$ in space. The numerical solution is compared with the analytical solution in the supremum norm. 


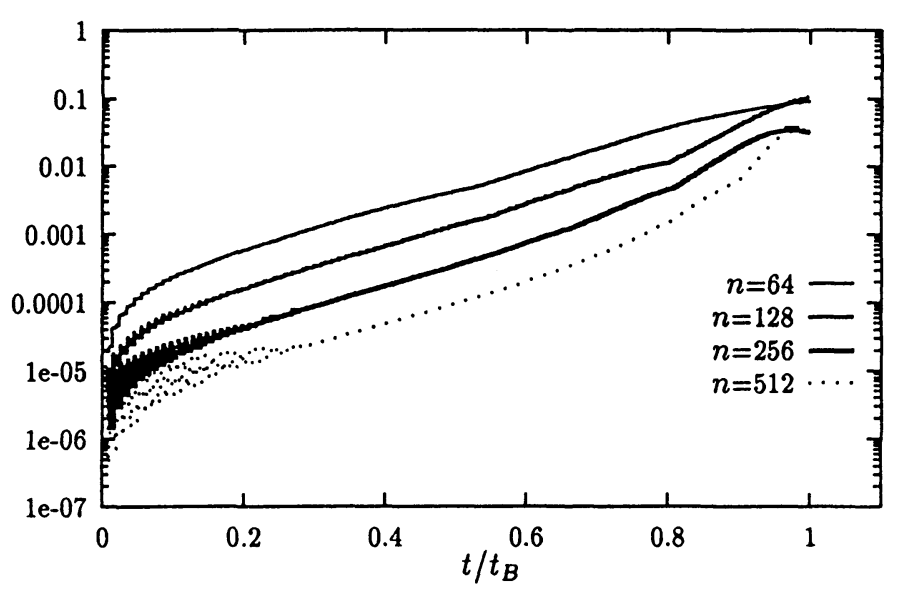

Fig. 2. The evolution of the error in the example shown in Fig. 1 for different discretizations. The error is the maximum of the relative error in $r$ and in each of the components $u^{a}$ and $h^{a}$

Our numerical results show that

(a) the scalar field $c_{1}=u^{c} h_{c}$ remains identically equal to zero (there is not even a round-off error);

(b) the error in the conserved quantity $h / r$ is in the order of machine round-off error $\left(\leq 10^{-9}\right)$;

(c) the maximum error between the numerical solution and the analytical solution decays quadratically with grid size, in agreement with the second order accuracy of the numerical scheme. This is shown in Fig. 2, where the evolution of the error is given for different numbers of grid points, $n$. This result holds true as long as the wave remains away from breaking. This error is also remarkably independent of $\eta=\Delta t / \Delta x, \eta \leq 1$, for velocities, $v$, satisfying $\Gamma \leq 10$. Significantly smaller timesteps are required for velocities with larger $\Gamma$.

In computations of Newtonian and relativistic wave breaking, the numerical results have been the same or better than as given in the observations (a)-(c) above.

Figure 2 also shows that the leapfrog Crank-Nicholson method the error exhibits an exponential growth as a shock develops. The method of Orszag and Tang $[25,10]$ restores linear error growth away from the moment of breaking. However, this introduces initial errors and fails to reduce the error. It should be mentioned that in these computations the error in the Riemann invariant $J=\lambda+\phi$ shows a linear growth in time, and, therefore remains order of magnitude smaller than the total error given in Fig. 2. Advanced numerical schemes should be used for solutions with shocks, as mentioned before.

Acknowledgements. The author expresses his gratitude to Prof. E. S. Phinney for his continued support during the course of this work. Discussions with Prof. T. de Zeeuw during the early stages of this work and critical comments by Mr. Eanna E. Flanagan, Mr. Tasso J. Kaper, and Prof. K. S. Thorne are also gratefully acknowledged. 


\section{References}

1. Anile, A.: Relativistic fluids and magneto-fluids. Cambridge: Cambridge University Press 1989

2. Anile, A., Bruhat, Y. (eds.): Lecture notes in mathematics: relativistic fluid dynamics. Berlin, Heidelberg, New York: Springer 1987

3. Harten, A.: Eno schemes with subcell resolution. J. Comp. Phys. 54, 148-184 (1984)

4. Lichnerowicz, A.: Etudes mathématique des fluides thermodynamique relativiste. Commun. Math. Phys. 1, 328-373 (1966)

5. Lichnerowicz, A.: Relativistic hydrodynamics and magnetodynamics. New York: Benjamin 1967

6. Lichnerowicz, A.: Shock waves in relativistic magnetohydrodynamics under general assumptions. J. Math. Phys. 17, 2135-2142 (1975)

7. Eringen, A.C., Maugin, G.A.: Electrodynamics of continua. II. Berlin, Heidelberg, New York: Springer 1990

8. Fischer, A.E., Marsden, J.E.: The einstein evolution equations as a first-order quasi-linear symmetric hyperbolic system. Commun. Math. Phys. 28, 1-38 (1972)

9. Carter, B., Gaffet, B.: Standard covariant formulation for perfect-fluid dynamics. J. Fluid. Mech. 186, 1-24 (1988)

10. Orszag, B., Tang, M.C.: Small-scale structure of two-dimensional magnetohydrodynamic turbulence. J. Fluid. Mech. 90, 129-143 (1979)

11. Choquet Bruhat Y., DeWitt-Morette, C., Dillard-Bleick, M.: Analysis, manifolds and physics. Part I: Basics. Amsterdam: North-Holland 1982

12. Evans, R.C., Hawley, J.F.: Simulation of magnetohydrodynamic flows: a constrained transport method. Astr. Ph. J. 332, 659-677 (1988)

13. Courant, R., Hilbert, D.: Partial differential equations. New York: Interscience 1967

14. Shu, C.W., Osher, S.: Efficient implementation of essentially nonoscillatory shock-capturing schemes ii. J. Comp. Phys. 54, 32-78 (1984)

15. Warner, F.W.: Founations of differential manifolds and Lie groups. Berlin, Heidelberg, New York: Springer 1983

16. Witham, G.R.: Linear and nonlinear waves. New York: Wiley 1974

17. Friedrichs, K.O.: On the laws of relativistic electro-magneto-fluid dynamics. Commun. Pure Appl. Math. 28, 749-808 (1974)

18. Misner, C.W., Thorne, K.S., Wheeler, J.A.: Gravitation. San Francisco, CA: Freeman 1973

19. Anile, A.M., Pennisi, S.: On the mathematical structure of test relativistic magnetohydrodynamics. Ann. Inst. H. Poincaré 46, 27-44 (1987)

20. Wald, R.M.: General relativity. Chicago, IL: Universtity of Chicago Press 1984

21. Muscato, O.: Breaking of relativistic simple waves. J. Fluid Mech. 196, 223-239 (1988)

22. Colella, P., Woodward, P.: The piecewise parabolic method (ppm) for gas-dynamical simulations. J. Comp. Phys. 54, 174-201 (1984)

23. Garabedian, P.: Partial differential equations. New York: Chelsea 1986

24. Woodward, P., Colella, P.: The numerical simulation of twodimensional fluid flow with strong shocks. J. Comp. Phys. 54, 115-173 (1984)

25. Peyret, R., Taylor, T.D.: Computational methods for fluid flow. Berlin, Heidelberg, New York: Springer 1983

26. Bruhat, Y.: Fluides relativiste de conductibilité infinite. Acta Astronautica 6, 354-363 (1960)

27. Bruhat, Y.: Etudes des équations des fluides relativistes charges et inductive conducteurs. Commun. Math. Phys. 3, 334-357 (1966)

28. Muller zum Hagen, H., Seifert, H.J.: On characteristic initial-value and mixed problems. General Relativity and Gravitation 8, 259-301 (1977) 
